\title{
Clase y género en las propuestas de la "Humanización" del parto: Un análisis desde la Antropología feminista
}

\author{
CELESTE JEREZ*
}

\begin{abstract}
Resumen
Actualmente existen distintos sentidos brindados al término "Humanización" dentro del ámbito del parto, que entran en diálogo y en disputa por un espacio político a ser ocupado. Algunos de estos sentidos son el resultado de los históricos reclamos y de la lucha de determinadas mujeres, de agrupaciones y de activistas del movimiento feminista. Este proceso aportó a la consolidación de las dos Leyes Nacionales sobre parto ( 25.929 y 26.485 , artículo 6 inciso "e"). Partiendo de una perspectiva feminista, en este artículo realizaré una lectura antropológica de la problematización social de los partos, incluyendo el análisis de los alcances de clase y de género que la "Humanización" del parto tiene como acto político de ciertas mujeres y agrupaciones.
\end{abstract}

Palabras claves: "Humanización" de los partos - Antropología feminista - clase y género

\begin{abstract}
Nowadays there are different meanings given to the term "humanization" of childbirths, which enter into dialogue and dispute over a political place to be occupied. Some of these meanings are the result of the historical claims and the struggle of certain women, groups and activists of the feminist movement over. This process contributed to the consolidation of the two national laws on childbirths $(25,929$ and 26,485 , Article 6, paragraph "e"). From a feminist perspective, in this article I will make a anthropological analysis of the social problematization of childbirths, including the class and gender scope of the "humanization" of childbirth as a political act of certain women and groups.
\end{abstract}

Key Words: "Humanization" of childbirths - Feminist Anthropology class and gender

Jerez, Celeste "Clase y género en las propuestas de la "Humanización" del parto: Un análisis desde la Antropología feminista", en Zona Franca. Revista del Centro de Estudios Interdisciplinario sobre Mujeres, Año XXII, No 23, 2014, pp. 83-92.

Recibido: 12 de agosto 2014 - Aceptado: 2 de octubre 2014 


\section{Introducción}

En los últimos 20 años, diversas autoras han resaltado el trato invasivo por parte del personal de la salud (DavisFloyd, 1993; Merino y Fornes, 2008; Canevari Bledel 2011), que tiene la característica de plasmarse por igual hacia todas las mujeres en el momento del parto. El reconocimiento de la violencia implícita en este tipo de trato, se vincula con prácticas que son efectuadas a las mujeres y específicamente a sus cuerpos, por estar agrupadas según determinadas características resaltadas, en este caso la posibilidad de parir. La atención biomédica del parto se convierte entonces en el evento a través del cual las mujeres son agrupadas y homogeneizadas (Tubert 1991; Jordan 1993; Sadler 2001, 2004; Wagner 2002; Blázquez Rodríguez 2005; Merino y Fornes 2008; Fornes 2011), en donde sus cuerpos son pasibles de prácticas por igual.

El evento del parto es entendido dentro del "Modelo Tecnocrático de Nacimiento" (Davis-Floyd; 1993; 2001) $)^{1}$ como un proceso únicamente fisiológi$\mathrm{co}$, donde pese al protagonismo del cuerpo de las mujeres, lo que ellas sienten, expresan y saben tiene poca o nula influencia sobre el proceso. Sin embargo, si bien se entienden los procesos de manera "fisiológica", subyacen todo tipo de evaluaciones simbólicas sobre los cuerpos de las mujeres. La mayoría de los profesionales de la salud dan por supuesto no solo que toda mujer debe ser madre como destino, sino que toda mujer desea ser madre. Se parte entonces de la idea de que las mujeres que no aman a sus hija/os transgreden mandatos sociales, así como "mandatos naturales" al asociar linealmente el ser mujer a maternidad y a naturaleza (Tarducci, 2008). En las instituciones por lo general no se asume que muchos embarazos pueden no ser deseados ni planeados y que pueden resultar traumáticos para algunas mujeres. Además, los profesionales de la salud suelen creer que específicamente las mujeres pobres no planifican a sus hija/os, y cuando sospechan que algún embarazo no es deseado, las mujeres reciben humillaciones (Canevari Bledel, 2011).

\section{“Lo personal es político": El parto como problema social}

Lejos de ser uniforme, la problematización de temáticas como el parto que en el pasado se creían de "la vida privada" de las mujeres, es puesta en práctica desde distintas conceptualizaciones que entran en disputa por un espacio político a ser ocupado. Igualmente, estas diversas narrativas se inscriben en una genealogía particular. ¿Quiénes reinterpretaron estos hechos, como la violencia ejercida en los partos, y posibilitaron nuevas narrativas partiendo de la violencia hacia las mujeres como un problema social? El movimiento feminista a través de múltiples formas de denuncias tales como elaboraciones teóricas y acciones concretas, fue construyendo marcos de referencia para interpretar ciertos hechos de la realidad como desiguales y violentos para las mujeres, y proclamando autonomía para ellas. Desde las primeras reivindicaciones feministas a principios del siglo $X X$ y a lo largo de toda su historia, el estrecho lazo entre teoría y política signó el curso de rumbo del movimiento feminista aunque con distintos efectos en cada etapa y en cada país (Tarducci y Rifkin, 2010). En este sentido, la relevancia de ciertos escritos teóricos promovió la consolidación de una "autonomía interpretativa" (Miguel Álvarez, 2003 ; 17) dentro del movimiento de mujeres, es decir una forma propia y específica de analizar la realidad. Como bien analiza Déborah Daich (2010) para la violencia doméstica, el hecho de denominar "violencia" a una serie de conductas y comportamientos que tradicionalmente se entendían como normales, forma parte del proceso de reconocimiento y conceptualización de la violencia. De esta manera, son determinadas narrativas que los sujetos sociales comienzan a elaborar las que permiten generar rupturas de sentido en la vida social. En mi caso de investigación, el hecho de denominar como violentas ciertas conductas y comportamientos naturalizados durante el proceso del parto, hizo que la sociedad civil comience a reconocer un problema en las prácticas obstétricas cotidianas.

Si consideramos las leyes como un "producto final, público y visible de un largo y complejo proceso de redefinición de la violencia contra las mujeres" (Miguel Álvarez, 2003; 5), significa entonces que tienen el peso de condensar procesos impulsados anteriormente. Por ello, es necesario destacar cómo organizaciones de la sociedad civil, entre ellas feministas, y otros actores construyeron sentidos de denuncia que luego se plasmaron en las dos leyes nacionales específicas sobre el parto (Leyes Nacionales 25.929 y 26.485, artículo 6 inciso "e").

Sin embargo, en nuestro país aunque la violencia obstétrica es desde hace 6 años objeto de reglamentación legal integrada como artículo de la ley que protege a las mujeres de la violencia de género ${ }^{2}$, existe desde 
hace 11 años una Ley Nacional sobre los derechos de las mujeres, hijas e hijos durante el parto, que aún no está reglamentada.

La ley Nacional $n^{\circ} 25.929$ sobre Derechos en el Nacimiento, también es Ilamada Ley de Derechos de Padres e Hijos durante el Proceso de Nacimiento y, de manera informal, "Ley de parto humanizado, parto respetado", y fue promulgada en septiembre del año 2004. Aunque aún no reglamentada, la misma se aplica para los ámbitos públicos y privados de la atención de la salud, mencionando en el artículo 2 los derechos específicos de las mujeres en relación con su embarazo, trabajo de parto, parto y postparto. Cabe resaltar el foco de este artículo puesto en la obligación del personal de la salud a informar sobre su accionar, es decir sobre las intervenciones médicas efectuadas a la mujer, sobre la evolución de su parto y el estado de su hija/o, y al derecho de la mujer a la elección cuando existieran diferentes alternativas. Lo mencionado se relaciona con otro inciso del artículo que focaliza en considerar a las mujeres en el proceso de parto como personas sanas, promoviendo su participación y protagonismo en este evento ${ }^{3}$. El último aspecto a resaltar de este artículo, es el foco puesto en el derecho de la mujer a estar acompañada por una persona de su confianza y elección durante el trabajo de parto, parto y post-parto ${ }^{4}$.

Actualmente en nuestro país, "parto humanizado/ respetado5", "violencia obstétrica" y, como veremos más adelante, "parto en casa", forman parte de una retórica particular que se inscribe en las narrativas sociales acerca de los derechos de las mujeres y de la violencia de género. Esta retórica genera rupturas de sentido en la vida social, donde ciertas prácticas naturalizadas en relación al parto son convertidas en problemas sociales (Jerez, 2015).

En mi tesis de Licenciatura en Ciencias Antropológicas, los textos de la antropóloga Valeria Fornes $(2009$; 2011) fueron claves para comenzar a analizar la existencia en América Latina de mujeres que logran "partos humanizados" en algunos hospitales específicos y en sus hogares, donde efectúan un "empoderamiento" de sus propios cuerpos, es decir, donde pueden ser concientes de su protagonismo al momento de parir, elegir a la/os acompañantes, ser ayudadas por parteras, parir sin demasiadas (o nulas) intervenciones médicas y experimentar espacios donde poder expresar deseos, angustias y sentimientos. Me encontré en el año 2012 con que existían a nivel nacional varias agrupaciones que promovían el "parto humanizado", como grupos de "partos en casa", militantes feministas, parteras organizadas, "grupos de crianza", mujeres independientes, entre otras. La Red Latinoamericana y del Caribe para la Humanización del Parto y el Nacimiento, RELACAHUPAN, desde principios del 2000 nuclea a muchas de estas agrupaciones y equipos, así como organiza todos los años, en la tercera semana del mes de mayo, la "Semana Mundial por el Parto Respetado" en nuestro país. A su vez, diversos actores fueron creando una "comunidad virtual" que se reúne diariamente a través de las redes sociales y los listados electrónicos. De esta forma, basados en el "Ideario de la Humanización" (Tornquist, 2003), mujeres usuarias, agrupaciones, agentes estatales, feministas, profesionales de la salud, terapeutas alternativos, ONG's, usuarias de redes vir- tuales, entre otros se relacionan y politizan los partos, la violencia y la maternidad. En este sentido, los referentes empíricos de mi tesis fueron dos agrupaciones que promovieran y/o militaran el "parto humanizado" o criticaran la violencia obstétrica: La Colectiva Maternidad Libertaria y Las Casildas, ambas de la Ciudad de Buenos Aires.

Al trabajar etnográficamente con estas dos agrupaciones que militan y/o promueven distintos enfoques de la "Humanización" del parto en nuestro país, intenté realizar una lectura antropológica de la problematización social de los partos de las mujeres. Partiendo de una perspectiva feminista dentro de la Antropología, en este artículo me focalicé en los alcances de clase y de género que la "Humanización" del parto tiene como acto político de ciertas mujeres y agrupaciones.

\section{La "Humanización del parto" como categoría polisémica}

El "Ideario de la Humanización" entendido como los valores, conceptos y prácticas que se proponen como base para la Humanización del parto, se refleja en las recomendaciones y directrices vigentes propuestas por la Organización Mundial de la Salud, que diferentes actores y organismos nacionales e internacionales han adoptado (Tornquist, 2003). Estas recomendaciones sintetizan una pluralidad de investigaciones sobre parto en el mundo entero.

Este "Ideario" tiene distintos significados dependiendo del actor que los proponga. Y aún más, estos diversos actores sociales se relacionan y entran en disputa por un espacio político a ser ocupado. En este sentido, el "Ideario de la Humanización" se pone en práctica a través de los diversos usos políticos que 
actores sociales, agrupaciones y sectores le dan al término. Cada sentido brindado al término "Humanización" se entiende como una "reivindicación de legitimidad del discurso" (Diniz, 2005), es decir, como reivindicaciones de diferentes sectores convergentes o contrapuestos, que entran en diálogo por este espacio político a ser ocupado. En este sentido, ¿puede una temática como el "parto humanizado" reflejar intereses y alcances de clase distintos? Es decir, ¿Qué alcances en términos de clase tiene cada sentido de "Humanización" de los partos brindado por esta diversidad de actores sociales? ¿A qué mujeres concretamente logran representar?

Para la comunidad médicocientífica, la "Humanización" resalta la asistencia basada en la evidencia científica. A través de revisiones sistemáticas de ensayos clínicos aleatorios, esta propuesta reivindica el uso apropiado de la tecnología y el respeto a la fisiología de las mujeres. A su vez, se opone a las prácticas guiadas por la opinión y por la tradición, apropiándose políticamente del discurso técnico. Por ejemplo, en "Episiotomía en el parto vaginal" (Carroli y Belizán, 2008) de la Biblioteca Cochrane $^{6}$, a través de la revisión de estudios clínicos controlados aleatorizados, evalúa los beneficios del uso restrictivo de la episiotomía en comparación con la episiotomía rutinaria durante el parto vaginal, que demuestra menos trauma perineal posterior, menos necesidad de sutura y, por consiguiente, menos complicaciones fisiológicas futuras para las mujeres.

En cambio, la asistencia de los partos basada en los derechos humanos enfatiza el rol de la "Humanización" en la defensa y reivindicación de los derechos de las mujeres (Diniz, 2005). Esta búsqueda de legitimidad política, a diferencia de la legitimidad científica reivindicada por el discurso anterior, busca una agenda política marcada por los derechos (no) reproductivos y sexuales, en el marco de los derechos sociales. Es en esta línea, aunque con usos particulares y con determinados límites, que ambas agrupaciones con las que he trabajado, la Colectiva Maternidad Libertaria y Las Casildas, construyen la "Humanización" del parto ligada a la promoción de los derechos de las mujeres. De aquí que ambas hayan participado en los últimos años de las movilizaciones que año a año rodean el Congreso de la Nación: el Día Internacional de las Mujeres (8 de Marzo) y el Día Internacional de la Eliminación de la Violencia contra la Mujer (25 de Noviembre).Por ejemplo, para estas ocasiones en el 2011 la Colectiva Maternidad Libertaria llevó remeras para vender con lemas de la temática del parto como "Ponele el cuerpo a tus derechos", esténciles con el lema "Todxs nacemos de mujer" y "Mi cuerpo, mi decisión", y una publicación con el lema "¡Parir con placer, Parir con poder!"7 que tenían para distribuir entre la/os presentes. A su vez, para el 25 de noviembre de aquél año, repartieron un "Test de Violencia Obstétrica" que tiene como encabezado el título "25 de Noviembre, Día Internacional de la Eliminación de la violencia contra la Mujer" y menciona el marco legal vigente para el parto (Leyes Nacionales 25.929 y 26.485 , artículo 6 inciso "e"). Son 12 preguntas cerradas que ahondan sobre la atención médica del parto y aborto en instituciones. Entiendo entonces que el Test actúa como un instrumento que refleja la "Humanización" del parto en término de derechos de las mujeres $y$, a su vez, marca una agenda política atravesada por los derechos reproductivos, sexuales y no reproductivos, en el marco de los derechos sociales. Por su parte, Las Casildas a partir del primer Encuentro Nacional de Mujeres al que asistieron en el 2012, y del trabajo con mujeres durante ese año, comenzaron a utilizar el término de "violencia obstétrica" y a dejar de hacer tanta referencia al "parto en casa", transición que se fue profundizando en los meses siguientes con las primeras referencias al feminismo ${ }^{8}$. Además, en sus redes virtuales comenzaron a armar "piezas virtuales", en palabras de una de mis entrevistadas, como las fotografías con inscripciones o spots fílmicos donde tenían como objetivo brindar información sobre las dos Leyes Nacionales existentes, en ocasiones puntuales como la Semana Mundial por el Parto Respetado o el Día Internacional de la Eliminación de la Violencia contra la Mujer. Específicamente, realizaron un trabajo en relación al inciso "g" del artículo 2 de la Ley Nacional 25.929, que aborda el derecho de la mujer a estar acompañada por una persona de su elección, reclamando por su reglamentación a través de fotografías y spots que circularon por las redes virtuales.

Por otro lado, utilizado en el lenguaje de las políticas públicas en el ámbito de la salud, la "Humanización" de la asistencia se plantea como el resultado de la tecnología apropiada para la salud de la población. Enfocándose en la asistencia de la población más que en la de individuos, se reivindica la racionalidad del uso de los recursos disponibles y apropiados. Este sentido brindado al término, puede verse reflejado en una iniciativa Maternidades Segu- 
ras y Centradas en la Familia (MSCF), que desde el $2010 \mathrm{im}$ plementa el actual Ministerio de Salud de la Nación, en conjunto con UNICEF y los ministerios provinciales, y que actualmente se lleva a cabo en 102 hospitales públicos del país ${ }^{9}$. Es interesante como éste sentido de la "Humanización" del parto puede yuxtaponerse (aunque con determinados límites) con el brindado tanto por el discurso de la evidencia científica antes mencionado, como con el de los derechos de las mujeres como derechos sociales.

Otro sentido otorgado al término es propuesto por los profesionales de la salud, orientado tanto en el cambio de jerarquías de los profesionales durante el parto como del escenario donde sucede. Si lo que prevalece actualmente es el "Modelo Tecnocrático de Nacimiento" es decir un modelo de atención donde la embarazada es una "pacienteenferma” (Davis-Floyd; 1993), son la/os medica/os obstetras quienes los atienden generalmente en quirófanos, por más que sean partos en condiciones "normales". Dada esta situación, si en la escena del parto están tanto obstetras como parteras/ licenciada/os en obstetricia, será la/el obstetra quien decida qué hacer, ya que ése es el escenario donde asume el poder frente a otros profesionales de la salud. De esta manera, la "Humanización" del parto relacionada al cambio de jerarquías de los profesionales y del escenario, vendría a discutir con el actual modelo. Las parteras y las enfermeras obstétricas pasarían a tener un lugar privilegiado en la atención institucional de los partos, así como las salas y casas de parto sustituirían a la sala quirúrgica. Este sentido dado al término "Humanización" es llevado actualmente a la prác- tica en algunas maternidades de Argentina y de Brasil.

Existe otro sentido de la "Humanización" de la asistencia en los partos, guiada esta vez por el discurso económico que evalúa costos y beneficios, que apunta a la racionalidad en el uso de los recursos materiales. Demostrando por ejemplo los altísimos costos que implican los diferentes "cortes", como las episiotomías, que se les realizan a las mujeres en situación de parto. Este discurso es clave para entender la relación económica entre clínicas privadas y el elevadísimo índice de cesáreas. Tuve una primera aproximación a este sentido de "Humanización" de la asistencia a los partos cuando comencé mi trabajo de campo en mayo del 2012 en la "Semana mundial por el parto respetado". Para esta ocasión la Red Latinoamericana y del Caribe por la Humanización del Parto y el Nacimiento (RELACAHUPAN) organizó unas jornadas en la Legislatura Porteña tituladas "Los nacimientos y la economía". En el panel de aquélla ocasión participaron María José Bernasconi, coordinadora nacional de la RELACAHUPAN, la doula Sonia Cavia, Carla Veleda de la agrupación Parteras Independientes y la periodista Luciana Peker. Sonia Cavia por ejemplo focalizó no solo en los altos costos que implican las cesáreas y episiotomías, sino también en los gastos públicos que comprende la curación psico-emocional de las mujeres durante el post parto. A su vez, la partera Carla Veleda, explicó la relación entre el "Modelo Tecnocrático de Nacimiento", las prácticas cortantes y sumamente costosas, y la hegemonía del/a médico/a obstetra en el escenario del parto. En este sentido, la partera puso en juego dos sentidos de
"Humanización" de la asistencia al parto; por un lado, la del bajo costo de las prácticas que realizan las parteras cuando atienden partos, a diferencia de los obstetras, y el cambio en la jerarquía de los profesionales en la asistencia.

Por otra parte, basados en la tradición liberal y de defensa de los derechos de los consumidores, como señala Diniz (2005), existen discursos sobre la "Humanización" que reivindican la participación de cada parturienta en la decisión sobre su salud. Entre estos discursos podríamos ubicar ciertas propuestas del "Parto en casa"10, y las reflexiones en relación al parto que resaltan el individualismo dentro del mercado. Por ejemplo en las páginas web de las agrupaciones de parteras que asisten partos domiciliarios, se realiza una breve descripción de las ventajas de este servicio que fomentaría la participación de las personas involucradas en el proceso de embarazo, parto y post-parto, donde se ofrecen los números de contacto para contratarlas y la posibilidad de atender mediante obras sociales pre-pagas ${ }^{11}$. En nuestra sociedad neo-liberal y de economía capitalista, determinados discursos enfatizan aspectos relacionados con la elección individual, como si se tratara de sujetos que están asilados de sus contextos y de las desigualdades de clase propiamente estructurales que genera el mismo mercado ${ }^{12}$. Resulta igualmente interesante, el hecho de que esta crítica al parto en casa ha sido apropiada y puesta en tensión por algunas defensoras del mismo. Por ejemplo en Junio del 2014, una integrante del elenco de la obra de teatro "Parirnos"13 de Las Casildas, escribió una reflexión que circuló por las redes virtuales a raíz de la nueva 
presentación del Proyecto de Ley para restringir las incumbencias de las parteras al ámbito público-institucional ${ }^{14}$, donde proponía: "Dicen que somos unas hippies locas, o unas ricas y famosas, que somos snob $y$ que esto es una moda. Dicen también que el parto domiciliario planificado solo sucede en Capital y que además facilita el robo de niñxs... Pero quienes elegimos casa y elegimos parteras sabemos que hablan desde el total desconocimiento, démosle cara a esta elección consciente y responsable, pongámosle rostro a este derecho a elegir cómo, dónde y con quién parir (....)"15. Luego de esta reflexión titulada "Las caras del parto en casa", se invitaba tanto a la participación en una Encuesta de Partos Planificados en Domicilio, de modalidad virtual ${ }^{16}$, como al envío de fotos a una casilla de correo electrónico donde aparecieran las personas implicadas en un parto domiciliario sosteniendo un cartel con la inscripción "yo parí en casa" y los datos del parto (año, nombres de la partera, lugar de residencia y características del parto). Con estas fotos se armó un video que también circuló por las redes sociales para apoyar el rechazo al Proyecto de Ley que restringe el accionar de las parteras. Estas reflexiones nos permiten entender que entre los discursos del "Parto en casa" existen tensiones y pujas por el reconocimiento de diversas realidades socio-económicas de quienes los han practicado. Sin embargo, aunque excede los límites de este artículo, cabe preguntarse de qué se está hablando cuando estas activistas hablan de "parto en casa", qué derechos se ven garantizados y cuáles se ponen en riesgo, y fundamentalmente los derechos de quiénes se están poniendo en riesgo: ¿es un parto domiciliario en una casilla de un asentamiento un "parto en casa"?, ¿es un parto domiciliario, en medio del campo, un "parto en casa"? Así pues, "parto en casa" es una categoría empírica que hace referencia a experiencias concretas y determinadas por las condiciones materiales de existencia, como las nombradas; no cualquier "casa" es un lugar posible donde puede practicarse un parto.

Una última aproximación a la "Humanización", siguiendo siempre a Diniz (2005), comprende el derecho al alivio del dolor. Este discurso guiado por la experiencia de instituciones que sufren la falta de recursos materiales, propone como "humanitarios" a los procedimientos clínicos como la anestesia peridural. A su vez, se enfoca en la premisa de que algunas drogas, medicamentos y prácticas en el pasado eran únicamente posibles en clases medias-altas. Ubicando al médico en un papel humanitario y rescatista, este discurso es factible de ser utilizado para justificar cualquier intervención médica sobre las mujeres.

Contamos entonces con diversos sectores, agrupaciones $y$ actores sociales que utilizando el término de "Humanización" para la asistencia a los partos, le otorgan sentidos distintos que se conjugan, se yuxtaponen y se contraponen, para darle legitimidad al discurso. Estos sentidos brindados a la "Humanización" del parto están en permanente construcción. Algunos de ellos, pueden ser costosos para las mujeres, teniendo en cuenta que es posible entrever, tanto una reproducción de ciertos mandatos médicos sobre los cuerpos de las mujeres como una selección (y omisión) de mujeres como destinatarias que tienen ciertas características de clase y de género. De esta forma, ¿cómo puede entender la idea sobre la "Humanización" del parto una mujer que ni siquiera tuvo la posibilidad de poder elegir la continuación de un embarazo no deseado? Mi interés como antropóloga feminista estuvo puesto no solo en los alcances de clase que tienen determinadas propuestas en relación a la "Humanización" del parto en un contexto de desigualdades sociales, sino también en cómo estas propuestas pueden plantearse desde una reivindicación integral de los derechos de las mujeres, focalizando en los sexuales, reproductivos pero especialmente en los no reproductivos.

\section{La "Humanización" del parto como reivindicación de los derechos integrales de las mujeres}

A través de los aportes de Mónica Tarducci (2008), exploré el peso que tiene la maternidad como institución y como mandato en los discursos sobre la "Humanización" del parto. ¿Cómo construyó la agrupación Colectiva Maternidad Libertaria sentidos en relación a la "Humanización" del parto focalizando en la reivindicación de derechos de las mujeres?

A mediados del 2013, la agrupación que en el 2011 había articulado con el taller de "Violencia y Adolescencia" que se desarrollaba en la Villa 21-24 del barrio de Barracas, logra volver a este barrio para concretar una actividad de "formación de doulas ${ }^{17 "}$ :

Cecilia: Pensábamos en una formación de 2 o 3 días intensa, sobre todo para ellas, para que una vecina por manzana sea madre o no vea serlo como su horizonte, maneje la informa- 
ción básica de derechos y sepa cómo acompañar (los partos). La idea es que ninguna mujer vaya sola a parir. (Las itálicas me pertenecen)

¿Qué significado tiene el objetivo de que ninguna mujer vaya sola a parir dentro de la propuesta de la "Humanización" del parto de la Colectiva? Con el foco de la actividad puesto en hacer valer el derecho de las mujeres a ser acompañadas por una persona de su elección mencionado en la ley Nacional $n^{\circ} 25.929$, la Colectiva convocó a la Asociación Civil Dando a Luz para colaborar con la organización de "la formación de doulas" que llevó el nombre de "Encuentros de Formación de Referentes Barriales en Maternidad y Derechos"18. A su vez, articularon con la Comisión de Derechos Humanos Villa 2124, con el Grupo de Mujeres de la Villa 21-24 y con el Espacio Creativo Musical Orilleros para la convocatoria a las mujeres. EI sentido de la actividad para la Colectiva tenía muchas aristas para analizar, como me lo explicaba una de ellas:

Daniela: Si el Hospital para las mujeres del barrio es un lugar deseable, no vamos a lograr que lo denuncien por violencia obstétrica, y porque (ellas) te lo decian así abiertamente, "yo no voy a denunciar el lugar donde después tengo que hacer el control pediátrico de mis hijos, que lo necesito para cualquier cosa, no lo voy a denunciar".

De esta forma, la Colectiva está marcando una agenda política signada por los derechos (no) reproductivos y sexuales, cuando plantea acciones para definir los límites de un problema social como tal. Es decir, cuando elige no trabajar a través de la denuncia por violencia obstétrica, teniendo en cuenta que el Hospital es un escenario transitado por las mujeres y sus familias más allá del parto, está delimitando cómo problematizar el parto y cuál es el sentido de la "Humanización" para este contexto. A su vez, cuando plantea que ninguna mujer vaya sola a parir está definiendo un problema social, el problema es que las mujeres vayan a parir solas. Y a este problema se le suma otro. La Ley Nacional 25.929, contiene el ya mencionado artículo 2, Inciso "g", que establece que el derecho de la mujer a estar acompañada, por una persona de su confianza y elección durante el trabajo de parto, parto y postparto, pero la misma aún no está reglamentada. Si el hecho de que las mujeres vayan solas a parir significa un problema social, y si la actual Ley que permitiría solucionar este aspecto del parto no está reglamentada, la Formación de Referentes está definiendo al parto "Humanizado" de un modo particular. Por un lado, a través de problematizar el acompañamiento de los partos, es decir de su dimensión psicoemocional, $y$, por otro, a partir de la reivindicación de derechos de las mujeres que aún no están reglamentados luego de 11 años de su promulgación.

De esta forma, la Colectiva brinda al término de la "Humanización" del parto un sentido claramente de defensa y reivindicación de los derechos de las mujeres. "Mi cuerpo, mi decisión", frase que llevan en banderas y en esténciles reiteradas veces, la cual es generalmente utilizada para reclamar por el aborto legal, y el Test de Violencia Obstétrica, enfocado en la atención médica del parto y aborto en instituciones, muestran una interesante conexión entre el aborto legal y la promoción del parto "Huma- nizado", como derechos de las mujeres. Marcando una agenda política que vele por los derechos sexuales, reproductivos y no reproductivos, en el marco de los derechos sociales, la Colectiva Maternidad Libertaria propone la temática del parto a partir de la representación integral de las mujeres, tanto de las que desean ser madres como de las que no es su intención. Así, el alcance de su militancia se relaciona con un significado de "Humanización" de la asistencia que incluya tanto a los partos como al derecho al aborto legal.

\section{Consideraciones finales}

En mi Tesis de Licenciatura, una de las preguntas principales giró en torno a cómo se conjugan estas retóricas y términos relacionados al parto dado nuestro contexto nacional de inexistencia de una ley que garantice el aborto legal, seguro y gratuito. Definitivamente este tipo de debates en relación a los derechos a decidir sobre el propio cuerpo pueden ser integrales, incluyendo la promoción de los derechos sexuales, reproductivos y no reproductivos. A partir de lo relatado, quiero enfatizar que la actividad de "Formación de Referentes" de la Colectiva Maternidad Libertaria plantea una integralidad de la dimensión del acompañamiento de los partos, cuando propone tanto que el ámbito donde este acompañamiento suceda sea en el Hospital del barrio, o sea un espacio de acceso universal a la salud, como cuando propone la temática del parto a partir de la representación de las mujeres tanto de las que desean ser madres como de las que no es su intención. Lo que está en juego es el significado del parto como un evento social. Y, en este sentido, la propuesta del acom- 
pañamiento de los partos en el Hospital público por mujeres (y no exclusivamente madres) que forman parte de los vínculos barriales, produce tres supuestos. El primero, un fortalecimiento y re-creación de los lazos sociales entre las mismas mujeres de la Villa 21-24, un barrio emergente de la Ciudad de Buenos Aires; el segundo, una transformación de los vínculos entre profesionales de la salud del Hospital público y las mujeres del barrio atendidas en él, teniendo en cuenta que este vínculo está históricamente signado por relaciones de poder pero también que este escenario es transitado por ellas y sus familias más allá del parto; y el tercero, una apuesta por los derechos no reproductivos en tanto se resalta el deseo de la no-maternidad como una opción y como una elección de las mujeres, y se las incluye como posibles acompañantes de partos. Es decir, partiendo de un claro sistema de desigualdades sociales que hace que no todas las personas accedan de igual forma a los bienes de consumo y a la garantía de los derechos sociales, es una apuesta de la
Colectiva el hecho de trabajar en un barrio emergente con esta temática y en los Hospitales públicos. A su vez, anclar en un aspecto de la ley 25.929 que aún no está reglamentada, fortalecer los lazos sociales entre las mismas mujeres del barrio -sean madres, no lo sean o no deseen serlo-, e incluir al aborto legal entre sus demandas, son decisiones políticas de la militancia de la Colectiva que dimensionan las perspectivas de clase y de género con las que trabajan para transformar la realidad cotidiana de las mujeres.

\section{Notas:}

* Profesora y Licenciada en Antropología (UBA). Colectiva de Antropólogas Feministas. Instituto Interdisciplinario de Estudios de Género, Facultad de Filosofía y Letras, Universidad de Buenos Aires. celestemjerez@gmail.com

1. Robbie Davis-Floyd, antropóloga socio-cultural estadounidense especializada en Antropología de la Reproducción, describe 3 modelos de asistencia al parto/nacimiento en Occidente: Tecnocrático, humanizado y holístico. La autora los resume en doce principios, los cuales difieren principalmente en la definición del cuerpo y su relación con la mente, y en este sentido, en las implicancias que tienen en la atención de la salud. Específicamente en el primero de estos Modelos, la autora describe la alta tecnocratización y medicalización de cualquier parto que llega a una institución (que claramente varía de acuerdo a los recursos materiales y de especialización que cada institución posee) como prácticas basadas en la idea del embarazo-parto como una patología y como un riesgo.

2. Ley Nacional 26485 , artículo 6 inciso "e".

3. A su vez, se puntualiza en el derecho de la mujer "a ser tratada con respeto, de modo individual y personalizado que le garantice la intimidad durante todo el proceso asistencial y tenga en consideración sus pautas culturales" (Ley Nacional $N^{\circ} 25.929$ Artículo 2- Definición).

4. Ley Nacional $\mathrm{N}^{\circ} \mathbf{2 5 . 9 2 9}$ artículo 2, inciso "g".

5. A lo largo de este artículo utilizaré como sinónimos los términos "Parto Humanizado" y "Parto Respetado", como son utilizados en la Ley Nacional 29.929, aunque estas dos expresiones tengan sentidos distintos en ciertos contextos y para determinados actores sociales.

6. La Biblioteca Cochrane es una colección de bases de datos sobre ensayos clínicos controlados en medicina y otras áreas de la salud. Es el resultado de la Colaboración Cochrane, una organización sin ánimo de lucro que reúne a un grupo de investigadores de ciencias de la salud de más de 11.500 voluntarios en más de 90 países que aplican un riguroso y sistemático proceso de revisión de las intervenciones en salud. Se actualiza cada tres meses y distribuye mediante suscripción anual en CD o a través de Internet.

7. La publicación posee en su portada la imagen del rostro de una mujer gritando y debajo del lema citado, aparece la consigna "Podemos ser mujeres sin ser madres, podemos ser madres sin parir, pero también podemos parir sin sufrir". En el interior de la publicación, figuran relatos acerca de partos clínico-hospitalarios de diversas mujeres, en los que se puntualiza tanto en la violencia obstétrica como en determinadas prácticas relacionadas a la "Humanización" del parto.

8. Estas referencias comenzaron a aparecer en el 2013 en el programa radial "Enredo en el Aire: El programa de Las Casildas. Frases como "mujer escucha, únete a la lucha"; "saquen sus rosarios de nuestros ovarios", eran utilizados para el comienzo de cada programa. Estos cambios en su discurso que se relacionan con sus actividades con mujeres y puntualmente a partir de los "diálogos" con el movimiento feminista, marcaron un rumbo distinto al que venían transitando relacionado con la promoción del "parto en casa". 
9. http://www.msal.gov.ar/prensa/index.php/noticias/noticias-de-la-semana/2713maternidades-seguras-y-centradas-en-la-familia-para-fortalecer-los-derechosde-las-mujeres-y-sus-bebes (Fecha de consulta: Junio de 2015).

10. Tomaré el término "Parto en casa" como sinónimo de "Parto domiciliario".

11. Un ejemplo concreto es la organización Tobi Natal que en su página web, donde está el contacto de referencia, figura la leyenda "Atendemos por Swiss Medical y otras pre-pagas" (http://www.tobinatal.com.ar/; fecha de consulta: Abril de 2015)

12. Creo que es reconociendo esta lógica del mercado neo-liberal, que debemos defender y promover las autonomías tanto en el campo de la salud, como en otros campos sociales. La ley Nacional $n^{\circ} 25.929$ hace referencia al derecho de la mujer a la elección cuando existieran diferentes alternativas sobre las intervenciones médicas a ser efectuadas durante el proceso de parto. Este discurso también reivindica la participación de cada parturienta en la decisión sobre su salud, pero no hace referencia al "consumo de servicios" para lograr acceder a ellas.

13. La obra cuenta con 4 actrices que relatan distintas experiencias de partos, desde "partos en casa" hasta partos institucionales, donde visibilizan tanto las prácticas del personal de la salud en relación al parto como las experiencias físicas y psico-emocionales que atraviesan las mismas mujeres en esta situación. El estreno de la obra fue en Mayo de 2014 y continuó una vez al mes durante todo el año. Tuvo lugar en teatros, en hospitales de distintos lugares de la Provincia de Buenos Aires y en la Facultad de Medicina de la UBA, en Noviembre, con la presencia del Centro de Estudiantes de Obstetricia. En el 2015 continúan con la obra, presentándola en distintos hospitales y clínicas del país.

14. A mediados del año 2012 una diputada (y médica anestesista) por el oficialismo de aquel momento, María Elena Chieno, presentó un proyecto de Ley ("Regulación de la Actividad Profesional de Obstetricia") para reformar las incumbencias de las licenciadas en obstetricia, que entre otros puntos proponía reducir sus labores al ámbito institucional, dotando de ilegalidad la atención de los partos planificados en los domicilios. Mujeres independientes, parteras y específicamente la agrupación "Asociación Argentina de Parteras Independientes", se nuclearon en la defensa de los partos domiciliarios o "partos en casa", convocando movilizaciones de rechazo, coordinando una encuesta sobre esta modalidad de partos y presentando en el INADI una denuncia contra la diputada. Luego de algunos meses (el 11/11/2012) lograron que la Cámara de Diputados de la Nación expidiera la siguiente modificación al Proyecto de Ley. Sin embargo, a principios del 2014 el Proyecto de Ley perdió estado parlamentario y un grupo de diputados lo volvió a presentar en mayo de 2014 (proyecto de ley 2895-D-2014) proponiendo nuevamente restringir las incumbencias de las parteras al ámbito público-institucional. Se formó entonces la "Asamblea Por el Derecho a Elegir como Parir" que nuclea a distintas mujeres, organizaciones y diputados.

15. El fragmento se titula "Las caras del parto en casa", https://www.facebook.com/ notes/violeta-osorio/las-caras-del-parto-en-casa/10152950579987576 (fecha de consulta: Abril de 2015).

16. Esta encuesta se presenta en la página de la agrupación "Por el derecho a elegir cómo parir", actores claves en el rechazo del Proyecto de Ley descripto.

17. "El término doula designaba en la Grecia antigua a la esclava que ayudaba a la señora a parir, en Brasil y en otros países de Latinoamérica las llaman acompañantes de parto. (...) La antropóloga Dana Raphael fue la primera en emplear el término doula para referirse a las mujeres experimentadas que ayudaban a las nuevas madres en la lactancia y crianza de sus bebés. Después el término se extendió a las ayudantes prenatales y durante el parto por investigadores médicos como M. Klaus y J. Kennell". (Silin, 2010; 5-6)

18. Es interesante justamente el cambio en los términos utilizados, pasando de "doula" a "referente barrial", donde el foco estuvo a su vez puesto en articular con la Ley Nacional vigente. La formación gratuita brindada apuntaba a que sean las mismas mujeres del barrio las que, formadas en un abordaje psico-emocional, pudieran acompañar a otras mujeres en situación de parto y hacer valer sus derechos. En este sentido, el alcance de la actividad tuvo el objetivo de trascender ciertas barreras de clase para lograr un parto más cercano al modelo de parto "Humanizado".

\section{Bibliografía:}

BLÁZQUEZ RODRÍGUEZ, Maribel (2005). "Aproximación a la Antropología de la Reproducción”, en AIBR. Revista de Antropología Iberoamericana, № 42, 1- 22.

CANEVARI BLEDEL, Cecilia. (2011). Cuerpos enajenados: experiencias de mujeres en una maternidad pública, Barco Edita, Santiago del Estero. 
CARROLI Guillermo y BELIZÁN José (2008). "Episiotomía en el parto vaginal" (Revisión Cochrane traducida), en La Biblioteca Cochrane Plus, Oxford, $\mathrm{N}^{\circ} 3$.

DAICH, Déborah (2010). "Narrativas sobre la violencia doméstica", en Familias, conflictos y justicia. Tesis doctoral. Buenos Aires: Facultad de Filosofía y Letras, Universidad de Buenos Aires.

DAVIS-FLOYD, Robbie (1993). "The technocratic model of birth". In Tower Hollis, S. et al. (ed.) Feminist theory in the study of folklore (297-326). University of Illinois Press.

DAVIS-FLOYD, Robbie (2001) "The technocratic, humanistic, and holistic paradigms of childbirth", International Journal of Gynecology \& Obstetrics, № 75, 5-23.

DINIZ, Carmen Simone Grilo (2005). "Humanização da assistência ao parto no Brasil: os muitos sentidos de um movimiento", en Ciênc saúde coletiva, № 10.3, 627-637.

FORNES, Valeria (2009). "Cuerpos, cicatrices y poder. Una mirada antropológica sobre la violencia de género en el parto", en Actas I Congreso Interdisciplinario sobre Género y Sociedad: debates y prácticas en torno a violencias de género, $1^{a}$ ed. Universidad Nacional de Córdoba.

FORNES, Valeria (2011). "Parirás con poder... (pero en tu casa). El parto domiciliario como experiencia política contemporánea". En Karina Felitti (Coordinadora) Madre no hay una sola. Experiencias de maternidad en la Argentina. Buenos Aires: Ediciones Ciccus

JEREZ, Celeste (2015). "Partos empoderados': Clase y género en la crítica a la violencia hacia las mujeres en los partos", en Actas XII Jornadas Nacionales de Historia de las Mujeres- VII Congreso de Estudios de Género. Centro Interdisciplinario de Estudios de Género, Facultad de Humanidades, Universidad del Comahue, Neuquén.

JORDAN, Brigitte (1993). Birth in four cultures, a crosscultural investigation of childbirth in Yucatan, Holland, Sweden and the United States. Illinois: Fourth Ed. Prospect Heights, Waveland Press.

MERINO, Lucía y FORNES, Valeria (2008). Gestar y parir espacios de género. Un abordaje cultural sobre la palabra, la experiencia y el poder en los modelos de parto. Trabajo final del curso de posgrado. Construcción de proyectos en Ciencias Sociales. Investigación cualitativa, acción social y gestión cultural. CAICYT- CONICET.

MIGUEL ÁLVAREZ, Ana (2003). "El movimiento feminista y la construcción de marcos de interpretación: el caso de la violencia contra las mujeres", en Revista Internacional de Sociología, №35, 7-30.

SADLER, Michelle (2001). "El nacimiento como acontecimiento médico", en Werkén, $N^{\circ} 2,113-124$.

SADLER, Michelle (2004). "Así me nacieron a mi hija". En Nacer, educar, sanar Miradas desde la antropología del género (15-66). Santiago de Chile: Catalonia.

SILIN, Jessica (2010). "De mujeres, matronas, doulas y partos", en II Congreso Virtual sobre Historia de las Mujeres. Recuperado en Abril de 2015 de: http:// www.revistacodice.es/publi_virtuales/ii_congreso_mujeres/comunicaciones/ JESSICASILIN.pdf

TARDUCCI, Mónica (Organizadora) (2008). Maternidades en el siglo XXI, Espacio Editorial, Buenos Aires.

TARDUCCI, Mónica y RIFKIN, Déborah (2010). "Fragmentos de historia del Feminismo en Argentina". En Sonia Santoro y Sandra Chaher (Compiladoras), Las palabras tienen sexo II. Herramientas para un periodismo de género. Buenos Aires: Artemisa Comunicación Ediciones.

TORNQUIST, Carmen Susana (2003). "Paradoxos da humanização em uma maternidade no Brasil", en Cad. Saúde Pública, N 19, Sup 2, 419-427.

TUBERT, Silvia (1991). Mujeres sin sombra: Maternidad y tecnología. Siglo XXI de España Editores, España.

WAGNER, Marsden (2002). "El nacimiento en el próximo milenio", en $1^{\circ}$ Congreso Internacional de parto y nacimiento en casa. Jerez de la Frontera, Cádiz.

\section{Documentos:}

Ley Nacional N²5.929 de Derechos de Padres e Hijos durante el Proceso de Nacimiento. Declaración de Interés del Sistema Nacional de Información Mujer, por parte del Senado de la Nación.

Ley Nacional N²6.485 de Protección Integral para Prevenir, Sancionar y Erradicar la Violencia contra las Mujeres en los ámbitos en que desarrollen sus relaciones interpersonales. Art. 6, inc. e) Violencia obstétrica. 